\title{
Coxsackievirus B4: a Undervalued Pathogen that Associated with Hand, Foot and Mouth Disease Outbreak
}

Jinbo Xiao

Chinese Center for Disease Control and Prevention https://orcid.org/0000-0003-0791-0923

Jianxing Wang

Shandong Center for Disease Control and Prevention

Yong Zhang ( $\square$ yongzhang75@sina.com )

Chinese Center for Disease Control and Prevention https://orcid.org/0000-0002-2692-5437

\section{Dapeng Sun}

Shandong Center for Disease Control and Prevention

\section{Huanhuan Lu}

Chinese Center for Disease Control and Prevention

\section{Zhenzhi Han}

Chinese Center for Disease Control and Prevention

\section{Yang Song}

Chinese Center for Disease Control and Prevention

\section{Dongmei Yan}

Chinese Center for Disease Control and Prevention

\section{Shuangli Zhu}

Chinese Center for Disease Control and Prevention

\section{Yaowen Pei}

Shandong Center for Disease Control and Prevention

\section{Wenbo Xu}

Chinese Center for Disease Control and Prevention

\section{Xianjun Wang}

Shandong Center for Disease Control and Prevention

\section{Original Article}

Keywords: coxsackievirus B4, hand, foot, and mouth disease, disease outbreak, genotype, evolutionary dynamics

Posted Date: February 17th, 2021

DOI: https://doi.org/10.21203/rs.3.rs-207417/v1

License: @ (i) This work is licensed under a Creative Commons Attribution 4.0 International License. Read Full License 


\section{Abstract}

Objectives: This study was conducted to discover the causes of Coxsackievirus B4 (CVB4) -induced hand, foot, and mouth disease (HFMD) outbreaks and its evolutionary characteristics.

Methods: In this study, we sequenced isolates obtained during the outbreak for comparative analyses with previously sequenced strains. Phylogenetic analysis and evolutionary dynamics were performed to illustrate the genetic characteristics of CVB4 in China and worldwide.

Results: The nucleotide sequence of CVB4 isolated during the outbreak in 2011 was more similar to that of CVB4 isolated in Shandong Province, China in 2010 (95.7-99.4\%) than to other CVB4 isolated in China (90.9-98.8\%). A phylogenetic analysis showed that CVB4 originated from a common ancestor in Shandong. CVB4 strains isolated worldwide could be classified into genotypes A-E according to the VP1 region. All CVB4 strains in China belonged to genotype E. The global population diversity of CVB4 fluctuated substantially over time, and CVB4 isolated from China accounted for a significant increase in diversity of CVB4. The average nucleotide substitution rate in VP1 of Chinese CVB4 $\left(5.20 \times 10^{-3}\right.$ substitutions/site/year) was slightly higher than that of global CVB4 (4.82 × 10-3 substitutions/site/year).

Conclusions: These findings explain both the 2011 outbreak and a global increase in CVB4 diversity. In addition to improving our understanding of a major outbreak, these findings provide a basis for the development of surveillance strategies.

\section{Introduction}

Coxsackieviruses belong to genus Enterovirus within the family Picornaviridae, which are small non-enveloped RNA viruses with a single-stranded positive sense genome of approximately 7500 nucleotides (nt). They are associated with various diseases, such as paralytic diseases, hand, foot, and mouth disease (HFMD), meningitis-encephalitis, myocarditis, febrile diseases, and respiratory infections [1-3].

According to their pathogenicity in suckling mice, coxsackieviruses can be divided into two groups: A and B [4]. Additionally, 27 serotypes have been reported, many of which are related to severe diseases [5, 6]. Many serotypes of coxsackievirus group A (CVA) can cause HFMD [7-10], particularly Coxsackievirus A6 (CVA6), Coxsackievirus A10 (CVA10), and Coxsackievirus A16 (CVA16). Coxsackievirus group B (CVB) is associated with a variety of diseases and is isolated at a relatively low rate; these viruses can induce severe central nervous system and heart-related diseases [11, 12], such as aseptic encephalitis, pleurodynia, type 1 diabetes, and viral myocarditis. These strains also cause respiratory infections, febrile diseases, and other mild diseases.

CVB has caused many disease outbreaks worldwide [13-15]. Coxsackievirus B3 (CVB3) and Coxsackievirus B5 (CVB5) are considered the main CVB serotypes causing HFMD and aseptic meningitis [16-18]. Surveillance data in China have shown that the incidence of HFMD in Shandong Province is high, including an outbreak in 2011. Coxsackievirus B4 (CVB4) infections were found in 21 patients with HFMD. There are few reports of HFMD outbreaks caused by CVB4 in China and elsewhere, which makes the study meaningful.

To date (Jan. 22,2020), 14 full-length genomic sequences and 209 CVB4 sequences (> 851 bp) containing VP1 region have been deposited in GenBank. The CVB4 prototype strain (X05690/JVB/New York/US/1951) was isolated from a specimen from the American Type Culture Collection in 1951 [19]. Its full-length genome is 7395 nucleotides (nt) including a 5'-untranslated region (5-UTR) (743 nt), 3'-UTR (100 nt), and open reading frame (ORF) (6552 nt). The ORF encodes a polyprotein of approximately 2183 amino acids. The sequence of the CVB4 prototype strain (X05690/JVB/New York/US/1951) is highly similar to that of the CVB3 prototype strain (Nancy/Connecticut/US/1949). 
CVB3 is a frequent cause of disease outbreaks; accordingly, studies of the evolution and clinical characteristics of CVB4 are needed [19].

In this study, the entire VP1 region of 35 CVB4 strains (21 strains isolated from patients and 14 strains from healthy individuals) were obtained. Fourteen CVB4 strains were isolated from HFMD surveillance specimens in Shandong Province in 2010, and 21 CVB4 strains were isolated from HFMD outbreak specimens in Shandong Province in 2011. All available CVB4 genome sequences were analyzed by a bioinformatics approach and analyzed with respect to clinical characteristics. Combined with HFMD surveillance results obtained before the outbreak, an epidemiological study of the HFMD outbreak caused by CVB4 in Shandong Province was performed.

\section{Materials And Methods}

\section{Ethical considerations}

This study did not involve any human experimentation. The main purpose of the study was to contribute to public health and the prevention of HFMD outbreaks caused by enteroviruses. Samples were obtained from the national laboratory network established by the National Laboratory for Poliomyelitis in the National Institute for Viral Disease Control and Prevention (IVDC) of the Chinese Center for Disease Control and Prevention (China CDC). Written informed consent for the use of clinical samples was obtained from the parents of all patients. Parents agreed to the use of specimens for research purposes and to the collection of samples by trained professional staff. This study was approved by the second session of the Ethics Review Committee of IVDC. All experimental protocols were approved by IVDC, and the methods were carried out in accordance with the approved guidelines.

\section{Sample collection and virus isolation}

In total, 197 stool samples from healthy children and 178 stool samples from patients with HFMD in an outbreak were collected in Shandong Province in 2010 and 2011, respectively. The diagnostic criteria were children with rashes and herpes on the hands, feet, and mouth accompanied by fever symptoms. Most patients had mild symptoms. A small number of patients with severe infections may develop aseptic meningitis, viral myocarditis, encephalitis, acute flaccid paralysis, and other symptoms, and further development may lead to cardiopulmonary insufficiency and eventual death $[20,21]$. The 375 specimens were processed according to the Polio Laboratory Manual (World Health Organization, Geneva, Switzerland). The processed samples were inoculated into human rhabdomyosarcoma provided by the WHO Global Poliovirus Specialty Laboratory in the United States. Continuous monitoring was performed and harvested cell cultures after the cells exhibited a complete EV-like cytopathic effect (CPE).

\section{Molecular typing and VP1 sequencing}

Viral RNA was extracted from the harvested cell culture using the QIAamp Viral RNA Mini Kit (Qiagen, Hilden, Germany). The One-step RT-PCR Kit (Perfect Real Time, TaKaRa, Dalian, China) was used for enterovirus-specific real-time polymerase chain reaction (real-time PCR) [22]. Enterovirus-positive specimens determined by real-time PCR were subjected to reverse transcription polymerase chain reaction (RT-PCR) using the PrimeScript One Step RT-PCR Kit Ver. 2 (TaKaRa) and primers E486/ E488, E490/E492, and E494/E496 were used to amplify the partial VP1 region [23]. The PCR product was purified using a QIAquick PCR Purification Kit (Qiagen), followed by sequencing using an ABI 3130 Genetic Analyzer (Applied Biosystems, Foster City, CA, USA). Sequences were analyzed using the EV Genotyping Tool and the BLAST server [24]. To obtain the entire VP1 sequence of CVB4, specific primers were designed (upstream primer, CVB4-F: GTTATAGTTCCAGCCGAAGCG; downstream primer, CVB4-R: CCACACACAACTCTGCCAATC). The PCR product was purified and sequenced.

\section{Bioinformatics Analysis}


A total of 209 CVB4 VP1 sequences were obtained from the GenBank database and Muscle (v3.8.31_i86linux32) was used to generate alignments $[25,26]$. After screening and removing low-quality sequences (incomplete VP1 region or sequencing error), they were combined with 35 entire VP1 sequences of CVB4 strains isolated from Shandong Province in 2010 and 2011 for a phylogenetic analysis. RAxML (v8.2.12) was used to construct maximum likelihood trees based on 215 complete VP1 coding regions of CVB4 [27]. Nucleotide substitution models were GTR+G, as calculated using ModelGenerator 0.85 [28]. Support was estimated from 1000 bootstrap replicates, and the results were visualized using FigTree (v1.4.4). Maximum likelihood trees based on sequence set were constructed using MEGA (v7.0) [29] with 1000 bootstrap replicates to verify the tree topology obtained using RAxML. According to phylogenetic trees, genotypes were defined using a 15-25\% divergence threshold for the VP1 coding region [30, 31] [32]. BioEdit (v7.0.9.0) was used to obtain nucleotide and amino acid sequence similarities [33].

To evaluate the global epidemic dynamics of CVB4 over time, 200 entire VP1 sequences of CVB4 containing time and geographic information were analyzed. Sequences were analyzed using BEAST (v1.8.4) [34-36]. The nucleotide substitution model was GTR+G, as calculated using ModelGenerator 0.85 . The optimal combination of molecular clock model and tree prior was determined. A Bayesian Markov chain Monte Carlo (MCMC) analysis was run for $2 \times 10^{8}$ generations to ensure the convergence of each parameter. The sampling frequency was set to $2 \times 10^{4}$ generations. Tracer (v1.6) was used to check convergence and optimize the molecular clock and tree prior and to determine the estimated sample sizes (ESS) [37]. The ESS value should be at least 200. To analyze the dynamics of population diversity, a Bayesian skyline plot based on 200 CVB4 entire VP1 sequences was constructed using Tracer [38]. TreeAnnotator (v1.8.4) was used to construct a Bayesian maximum clade credibility (MCC) tree. The first $10 \%$ of sampled trees were removed with the burn-in option. The phylogenetic tree was visualized using FigTree.

In total, 70 VP1 sequences of CVB4 strains isolated from China were selected for an analysis of population dynamics using BEAST. The strict clock model and the Bayesian skyline tree prior were used. A Bayesian MCMC was run for $3 \times 10^{8}$ generations to ensure that each parameter can converge. The sampling frequency was set to $3 \times 10^{4}$ generations. Tracer was used to evaluate parameters. An MCC tree was constructed using TreeAnnotator. The burn-in option was used to remove the first $10 \%$ of sampled trees. FigTree was used to visualize the phylogenetic tree. Nucleotide and amino acid sequence similarities for comparisons between VP1 sequences of CVB4 strains isolated from Shandong Province in 2011 and other CVB4 VP1 sequences were estimated using BioEdit.

\section{Results}

\section{Summary of CVB4 data}

During the 2011 HFMD outbreak in Shandong Province of China, 178 children were infected, including 25 severe cases (14.0\%). Most children (76.4\%) were under 6 years of age. In specimens from infected children, 122 (68.5\%) enterovirus strains were detected in the laboratory. Among these, 31 (17.4\%) were coxsackievirus B strains and 21(11.8\%) were CVB4 (isolated from different cases), accounting for $67.7 \%$ of all detected Coxsackievirus B strains (Fig. 1).

Among 197 specimens from healthy children, 136 (69.0\%) were enterovirus-positive of which 20 (10.2\%) were identified as Coxsackievirus group B. Additionally, 14 strains were identified as CVB4, accounting for $70.0 \%$ of Coxsackievirus B detected in the study (Fig. 1).

\section{Global CVB4 genotype distribution}

In addition to 35 entire VP1 sequences of CVB4 obtained in Shandong Province in 2010 and 2011, 209 CVB4 sequences containing VP1 region were obtained from GenBank. Among the sequences obtained in GenBank, 29 did not meet quality control thresholds and 180 sequences were used for analyses. These isolates were from Australia ( $N=10), C a n a d a(N=$ 
1), China $(\mathrm{N}=35)$, Denmark ( $=17)$, Finland $(\mathrm{N}=20)$, France $(\mathrm{N}=12)$, India $(\mathrm{N}=7)$, Italy $(\mathrm{N}=1)$, Latvia $(\mathrm{N}=1)$, Madagascar $(\mathrm{N}=20)$, Netherlands $(\mathrm{N}=32)$, Pakistan $(\mathrm{N}=3)$, Poland $(\mathrm{N}=4)$, Romania $(\mathrm{N}=2)$, Russia $(\mathrm{N}=2)$, Turkey $(\mathrm{N}=$ 1), United Kingdom $(N=1)$, United States $(N=9)$, and unknown areas $(N=2)$, representing a wide distribution (Table 1). $A$ phylogenetic tree based on all entire VP1 sequences showed that CVB4 can be divided into genotypes $A, B, C, D$, and $E$ (Fig. 2). Genotype A included the prototype strain (X05690/JVB/New York/US/1951) and the other two CVB4 strains (GenBank accessions S39291 and DQ480420). Genotype B-D contain strains from several countries other than China. Genotype E consisted of CVB4 strains isolated from China $(N=70)$ and Australia $(N=3)$. CVB4 strains isolated from China were all classified as genotype $\mathrm{E}$, including the new sequences obtained in this study. CVB4 in China formed essentially an independent lineage in the phylogeny. Genotype E was the most prevalent CVB4 genotype in China. Basic data for each genotype are summarized in Table 1. 
Table 1

Nucleotide sequence similarity, amino acid sequence similarity, and basic information for entire VP1 region sequences of CVB4 genotypes.

\begin{tabular}{|c|c|c|c|c|c|c|}
\hline \multirow[t]{2}{*}{ Genotype } & \multirow{2}{*}{$\begin{array}{l}\text { GenBank accession } \\
\text { number }\end{array}$} & \multicolumn{2}{|l|}{ Identity in VP1 } & \multirow{2}{*}{$\begin{array}{l}\text { Isolated } \\
\text { country }\end{array}$} & \multirow[t]{2}{*}{ Year } & \multirow{2}{*}{$\begin{array}{l}\text { Intra-group } \\
\text { distances(\%) }\end{array}$} \\
\hline & & $\begin{array}{l}\text { Nucleotide } \\
\text { sequences(\%) }\end{array}$ & $\begin{array}{l}\text { Amino acid } \\
\text { sequences(\%) }\end{array}$ & & & \\
\hline$A$ & $\begin{array}{l}\text { X05690, S39291, } \\
\text { DQ480420 }\end{array}$ & $99.4-99.7$ & $99.2-100.0$ & USA, Italy & 1951,2006 & 0.3 \\
\hline $\mathrm{B}$ & $\begin{array}{l}\text { AF160048- AF160073, } \\
\text { GU142875 } \\
\text { KC558560- KC558573, }\end{array}$ & $83.3-100.0$ & $96.4-100.0$ & $\begin{array}{l}\text { Australia, } \\
\text { Canada, } \\
\text { Denmark, } \\
\text { Finland, } \\
\text { Netherlands, } \\
\text { USA }\end{array}$ & $\begin{array}{l}1959- \\
1997\end{array}$ & 11.8 \\
\hline $\mathrm{C}$ & AF159998- AF160007, & 86.0-99.8 & $97.5-100.0$ & $\begin{array}{l}\text { Finland, } \\
\text { France, } \\
\text { Netherlands }\end{array}$ & $\begin{array}{l}1964- \\
1996\end{array}$ & 11.5 \\
\hline D & $\begin{array}{l}\text { AF160008-AF160047, } \\
\text { AY919465, FJ868288, } \\
\text { FJ868289, FJ868330- } \\
\text { FJ868332, GU142877, } \\
\text { HF948089, } \\
\text { HF948090,JX513577, } \\
\text { JX513579-JX513581, } \\
\text { JX513583, } \\
\text { KF177118,KF412921, } \\
\text { KR232695, KR232711, } \\
\text { KR232712, KR232730- } \\
\text { KR232732, KR232739, } \\
\text { KR232743, KR232751, } \\
\text { KR232758, KR232760, } \\
\text { KR232761, KR232763, } \\
\text { KR232775, KR232776, } \\
\text { KR232778, KR232782, } \\
\text { KR232792, KR232793, } \\
\text { KR232796, KU189236, } \\
\text { KU189240, KU189242, } \\
\text { KU189251, KU841463, } \\
\text { KU841464, KY369904, } \\
\text { LS451289, MG451808, } \\
\text { MG845888,MG845889, } \\
\text { MK044541 }\end{array}$ & $83.9-100.0$ & $96.4-100.0$ & $\begin{array}{l}\text { Australia, } \\
\text { Finland, } \\
\text { France, } \\
\text { India, Latvia, } \\
\text { Madagascar, } \\
\text { Netherlands, } \\
\text { Pakistan, } \\
\text { Poland, } \\
\text { Romania, } \\
\text { Russia, } \\
\text { Turkey, UK, } \\
\text { USA }\end{array}$ & $\begin{array}{l}1977- \\
2016\end{array}$ & 11.5 \\
\hline E & $\begin{array}{l}\text { JF794741, JN016524, } \\
\text { JX308222, KC867091- } \\
\text { KC867093, } \\
\text { KF781519-KF781525, } \\
\text { KJ933328, KJ933329, } \\
\text { KM890276, KP289433, } \\
\text { KU566507, KX752784, } \\
\text { LC412980, MF179585- } \\
\text { MF179588, } \\
\text { MF422558-MF422563, } \\
\text { MF678139, MF678300, } \\
\text { MF678347, } \\
\text { MG922519-MG922523 }\end{array}$ & $88.7-100.0$ & $96.8-100.0$ & $\begin{array}{l}\text { Australia, } \\
\text { China }\end{array}$ & $\begin{array}{l}2007- \\
2017\end{array}$ & 8.0 \\
\hline
\end{tabular}


With an uncorrelated relaxed molecular clock model (exponential) and exponential growth tree prior, MCC trees based on sequences of 200 globally distributed CVB4 were obtained using BEAST (Fig. 3). The average nucleotide substitution rate for the VP1 coding region in all CVB4 strains worldwide was $4.82 \times 10^{-3}$ substitutions per site per year ( $95 \%$ highest posterior density (HPD): $4.22 \times 10^{-3}, 5.46 \times 10^{-3}$ ). The estimated coefficient of variation was 0.8925 (95\% HPD: 0.8151 , 0.9709), supporting the use of the uncorrelated relaxed molecular clock. The topological structure of the MCC tree constructed using BEAST was nearly identical to that of the ML tree constructed using RAxML. Global CVB4 strains isolated since 1930 formed two branches. Branch 1 contained genotype A arising in 1950 (including the prototype strain X05690/JVB/New York/US/1951) and genotype E arising in 2001. This branch included mainly Chinese and Australian strains. Branch 2 included genotype B (differentiated in 1951), genotype C (differentiated in 1959), and genotype D (differentiated in 1970). Isolates on this branch were geographically widespread (Oceania, North America, Asia, Europe, and Africa). CVB4 strains were continuously detected in many European countries, including Finland, France, and the Netherlands, since 1951, indicating that the strain was responsible for many epidemics with a long history in Europe. Based on the MCC tree and previous studies, CVB4 was prevalent in Madagascar in 2011, with 20 strains. Seven CVB4 strains were detected in children with diarrhea in India from 2005 to 2011 [39]. Additionally, 21 CVB4 strains were detected from specimens from the HFMD outbreak in Shandong Province in our study, indicating that CVB4 is prevalent worldwide.

According to a Bayesian skyline plot of the entire CVB4 VP1 sequence (Fig. 3), the global population diversity of CVB4 exhibited five changes over time. The first change was detected in 1945-1984, during which diversity increased. In 19451978, the growth rate was slow, and genotypes A, B, C, and D formed. During this period, CVB4 was mainly prevalent in the Netherlands and the United States. In 1979-1984, genotype B increased rapidly and appeared in Europe, Canada, and Australia. The second major change occurred in 1985-1989, when population diversity began to decline, possibly due to a global decline in the prevalence of genotype D. The third change occurred in 1990-1991, when the number of populations increased briefly, probably due to the global spread of genotypes B and D. In the fourth stage, 1992-2010, the population of CVB4 showed a slow decline, followed by a rapid decline. Isolates mainly belonged to genotype $D$ during the first half of this stage and to genotype E, mainly in China, in the second half of the stage. In the fifth stage, beginning in 2011, the diversity of CVB4 increased rapidly and then remained steady. During this period, there were many epidemics and outbreaks of CVB4, which may be related to the rapid increase in diversity. Gradual stabilization since then may be due to a decrease in the quantity of CVB4 found in disease surveillance.

\section{Phylogenetic analysis of CVB4 in China}

A total of 70 CVB4 strains isolated from China were analyzed using BEAST with strict molecular clock model and Bayesian skyline tree prior (Fig. 4). The average nucleotide substitution rate of the CVB4 VP1 coding region in China was $5.20 \times 10^{-3}$ substitutions per site per year ( $95 \%$ HPD: $\left.3.69 \times 10^{-3}, 6.78 \times 10^{-3}\right)$, which was slightly faster than that of the VP1 coding region in global CVB4 strains. The CVB4 strains isolated in China arise in 1995 and were detected between 2007 and 2014. The areas of monitoring covered Shandong Province, Inner Mongolia Autonomous Region, Fujian Province, Yunnan Province, and other regions in China. According to the Bayesian skyline plot of the entire VP1 sequence of CVB4, the change in CVB4 diversity in China can be divided into two stages. The decline before 2010 and rapid increase in diversity after 2011 suggest that the outbreak of HFMD in Shandong Province was an important determinant of the increase in CVB4 diversity in China.

The MCC tree showed that CVB4 strains isolated from the HFMD outbreak in Shandong Province in 2011 and a portion of CVB4 strains isolated from healthy individuals in 2010 diverged from a common ancestor (with a posterior probability of up to 0.89). The nucleotide sequence similarity between VP1 sequences of CVB4 in Shandong in 2011 was $99.6-100 \%$ and the amino acid sequence similarity was $100 \%$. The nucleotide sequence similarity in comparison with CVB4 strains isolated in Shandong Province in 2010 was $95.7-99.4 \%$ and amino acid sequence similarity was $97.8-98.9 \%$. The 
nucleotide sequence similarity with other CVB4 isolates from China was $90.9-98.8 \%$ and amino acid sequence similarity was $97.5-99.2 \%$.

\section{Discussion}

Frequent outbreaks of HFMD worldwide, disproportionately affecting children under 5 years of age, are a major public health issue. If patients are not treated in time, infant death may occur [40-43]. The main causal pathogens are EV-A71, CVA16, CVA6, and CVA10 [44-47]. CVB3 and CVB5 are related to HFMD and aseptic meningitis [16, 18]. CVB4 rarely causes HFMD outbreaks. Far fewer CVB4 sequences are available in GenBank compared with other enteroviruses, limiting studies of CVB4 and explaining the lack of routine monitoring.

In this study, fourteen CVB4 strains were isolated from healthy children in Shandong Province in 2010, and 21 CVB4 strains were isolated from children with HFMD in Shandong Province in 2011 . CVB4 accounted for $70.0 \%$ and $67.7 \%$ of total CVB in 2010 and 2011, respectively, and the proportion of strains classified as CVB4 far exceeded CVB3 and CVB5 causing an outbreak of aseptic meningitis. CVB4 detected in this outbreak clustered together in an MCC tree and all belonged to the predominant genotype in China (i.e., genotype E). Additionally, using 35 newly obtained CVB4 VP1 sequences and 180 CVB4 VP1 region sequences in GenBank, we identified five distinct CVB4 genotypes, A-E. CVB4 formed two main branches since 1930. Along branch 1, genotypes A and E arise in 1950. Genotype A included the prototype strain (X05690/JVB/New York/US/1951), and genotype E mainly included CVB4 strains isolated from China. CVB4 assigned to branch 2 differentiated into genotypes B, C, and D beginning in 1945. This branch includes isolates from many areas (such as Oceania, North America, Asia, Europe, and Africa), indicating that evolution in this lineage was complex. Our findings provide an overview of CVB4 evolution with wide coverage and multiple genotypes.

We further found that the global population diversity experienced three periods of growth. In the first period (1945-1984), CVB4 gradually increased and was prevalent in Europe, North America, and Oceania. In the second period, 1990-1991, diversity showed a temporary upward trend. The global prevalence of genotype B and D increased, explaining the overall increase in diversity. In the third period, beginning in 2011, a sharp and steep rise in diversity was detected. During this period, there were many outbreaks of CVB4, including the outbreak in Shandong Province evaluated in this study, which may explain the general increase in diversity. More than half a century of evolution has allowed CVB4 to spread and cause an epidemic, dominated by genotypes $D$ and $E$. Decreases in other genotypes may be attributed to a lack of clinical symptoms or inadequate monitoring. These genotypes have been reported in Europe, North America, Africa, Australia, and other regions, and monitoring should be strengthened, particularly since extant genotypes have the potential ability to cause disease outbreaks.

The average nucleotide substitution rate of the CVB4 VP1 coding region was higher than those of genotypes $\mathrm{B}$ and $\mathrm{C}$ of EV-A71 VP1 (4.6 $\times 10^{-3}$ substitutions/site/year). Additionally, the substitution rate of China CVB4 was slightly higher than that of the global CVB4 VP1 coding region. These results indicate that in China, CVB4 is active and has a high mutation rate; this may also explain the outbreak of HFMD in Shandong Province. Furthermore, current strains of CVB4 in China are genotype E. The strain responsible for the outbreak of CVB4 in Shandong Province is derived from local CVB4 and not from abroad. Some CVB4 strains isolated from healthy children in 2010 were closely related to CVB4 strains isolated from HFMD samples from the outbreak in 2011. They shared a common ancestral sequence and exhibited higher nucleotide sequence similarity with each other than with other CVB4 strains isolated in China at other times. These results indicate that the outbreak of HFMD caused by CVB4 in Shandong Province in 2011 could be attributed to a pathogen that existing in the population as early as 2010. Eventually, an outbreak occurred in Shandong in 2011, proving that CVB4 has the ability to cause an outbreak but usually causes a subclinical or asymptomatic infection in the healthy population. 


\section{Conclusions}

Our analysis of 35 entire VP1 sequences of CVB4 strains isolated in this study combined with CVB4 data available in GenBank provided insight into the global phylogenetic and epidemic characteristics of CVB4. After more than half a century, co-circulation of genotypes $D$ and $E$ resulted in the reported epidemics and outbreaks $[48,39]$. The outbreak of HFMD caused by CVB4 in Shandong Province is an example. These results broaden the pathogenic spectrum of HFMD. In addition, CVB4 isolated from the healthy population in 2010 was closely related to CVB4 isolated from patients with HFMD in 2011, indicating that the strain spread in the healthy population before the outbreak. When rare pathogens exhibit a sudden increase in the healthy population, careful monitoring is needed owing to the risk of HFMD or other diseases.

\section{Declarations}

\section{Ethics approval and consent to participate}

This study was approved by the Ethics Review Committee of the National Institute for Viral Diseases Control and Prevention, Chinese Center for Disease Control and Prevention. Written informed consent for the use of clinical samples was obtained from all individuals, including written informed consent from guardians of children.

\section{Consent to participate}

Written informed consent was obtained from the parents.

\section{Consent for publication}

Not applicable.

\section{Availability of data and materials}

The 35 CVB4 VP1 sequences determined in this study have been deposited in GenBank under accession numbers MT109013-MT109047.

\section{Competing interests}

The authors declare that no competing interests exist.

\section{Funding}

This study was supported by the National Key Technology R\&D Program of China (Project Nos. 2017ZX10104001, 2018ZX10711001, and 2018ZX10713002). The funding body was not involved in the design of the study, clinical sample collection, data analysis and interpretation, or writing of the manuscript.

\section{Authors' contributions}

JX studied the pathogens, analyzed the critical bioinformation, drafted the manuscript, and prepared all the tables and figures. JW investigated the outbreak, collected specimens, and identified the pathogens. YZ and XW conceived and designed the experiments, supervised and guided the experiments, and revised the manuscript. DS, HL, ZH, YS, DY, and SZ conducted part of the experiments. YP and WX analyzed the data. All authors read the manuscript.

\section{Acknowledgments}


We would like to acknowledge the staff of the Shandong Center for Disease Control and Prevention for collecting clinical samples.

\section{Code availability}

Not applicable.

\section{References}

1. Walker PJ, Siddell SG, Lefkowitz EJ, Mushegian AR, Dempsey DM, Dutilh BE et al. Changes to virus taxonomy and the International Code of Virus Classification and Nomenclature ratified by the International Committee on Taxonomy of Viruses (2019). Arch Virol. 2019;164(9):2417-29. doi:10.1007/s00705-019-04306-w.

2. Zell R, Delwart E, Gorbalenya AE, Hovi T, King AMQ, Knowles NJ et al. ICTV Virus Taxonomy Profile: Picornaviridae. J Gen Virol. 2017;98(10):2421-2. doi:10.1099/jgv.0.000911.

3. Sin J, Mangale V, Thienphrapa W, Gottlieb RA, Feuer R. Recent progress in understanding coxsackievirus replication, dissemination, and pathogenesis. Virology. 2015;484:288-304. doi:10.1016/j.virol.2015.06.006.

4. Crowell RL, Landau BJ. A short history and introductory background on the coxsackieviruses of group B. Curr Top Microbiol Immunol. 1997;223.

5. Gonzalez G, Carr MJ, Kobayashi M, Hanaoka N, Fujimoto T. Enterovirus-Associated Hand-Foot and Mouth Disease and Neurological Complications in Japan and the Rest of the World. Int J Mol Sci. 2019;20(20). doi:10.3390/ijms20205201.

6. Angez M, Shaukat S, Zahra R, Alam MM, Sharif S, Khurshid A et al. Characterization of group B coxsackieviruses isolated from non-polio acute flaccid paralysis patients in Pakistan: vital assessment before polio eradication. Epidemiol Infect. 2017;145(12):2473-81. doi:10.1017/S0950268817001522.

7. Wang M, Li J, Yao M-X, Zhang Y-W, Hu T, Carr MJ et al. Genome Analysis of Coxsackievirus A4 Isolates From Hand, Foot, and Mouth Disease Cases in Shandong, China. Front Microbiol. 2019;10:1001. doi:10.3389/fmicb.2019.01001.

8. Tan X, Li L, Zhang B, Jorba J, Su X, Ji T et al. Molecular epidemiology of coxsackievirus A6 associated with outbreaks of hand, foot, and mouth disease in Tianjin, China, in 2013. Arch Virol. 2015;160(4):1097-104. doi:10.1007/s00705-015-2340-3.

9. Yang Q, Zhang Y, Yan D, Zhu S, Wang D, Ji T et al. Two Genotypes of Coxsackievirus A2 Associated with Hand, Foot, and Mouth Disease Circulating in China since 2008. PLoS One. 2016;11(12):e0169021. doi:10.1371/journal.pone.0169021.

10. Tian H, Zhang Y, Sun Q, Zhu S, Li X, Pan Z et al. Prevalence of multiple enteroviruses associated with hand, foot, and mouth disease in Shijiazhuang City, Hebei province, China: outbreaks of coxsackieviruses a10 and b3. PLoS One. 2014;9(1):e84233. doi:10.1371/journal.pone.0084233.

11. Tracy S, Höfling K, Pirruccello S, Lane PH, Reyna SM, Gauntt CJ. Group B coxsackievirus myocarditis and pancreatitis: connection between viral virulence phenotypes in mice. J Med Virol. 2000;62(1):70-81.

12. Tam PE. Coxsackievirus myocarditis: interplay between virus and host in the pathogenesis of heart disease. Viral Immunol. 2006;19(2):133-46.

13. Verma NA, Zheng XT, Harris MU, Cadichon SB, Melin-Aldana H, Khetsuriani $\mathrm{N}$ et al. Outbreak of life-threatening coxsackievirus B1 myocarditis in neonates. Clin Infect Dis. 2009;49(5):759-63. doi:10.1086/605089.

14. Goldwater PN. Immunoglobulin M capture immunoassay in investigation of coxsackievirus B5 and B6 outbreaks in South Australia. J Clin Microbiol. 1995;33(6):1628-31.

15. Tao Z, Song Y, Li Y, Liu Y, Jiang P, Lin X et al. Coxsackievirus B3, Shandong Province, China, 1990-2010. Emerg Infect Dis. 2012;18(11):1865-7. doi:10.3201/eid1811.120090.

Page $10 / 16$ 
16. Han Z, Zhang Y, Huang K, Wang J, Tian H, Song Y et al. Two Coxsackievirus B3 outbreaks associated with hand, foot, and mouth disease in China and the evolutionary history worldwide. BMC Infect Dis. 2019;19(1):466. doi:10.1186/s12879-019-4107-z.

17. Liu N, Jia L, Yin J, Wu Z, Wang Z, Li P et al. An outbreak of aseptic meningitis caused by a distinct lineage of coxsackievirus B5 in China. Int J Infect Dis. 2014;23:101-4. doi:10.1016/j.ijid.2014.02.005.

18. Ma H, Huang X, Kang K, Li X, Tang X, Ren Y et al. Recombination in human coxsackievirus B5 strains that caused an outbreak of viral encephalitis in Henan, China. Arch Virol. 2013;158(10):2169-73. doi:10.1007/s00705-013-1709-4.

19. Jenkins O, Booth JD, Minor PD, Almond JW. The complete nucleotide sequence of coxsackievirus B4 and its comparison to other members of the Picornaviridae. J Gen Virol. 1987;68 ( Pt 7):1835-48.

20. Li X-W, Ni X, Qian S-Y, Wang Q, Jiang R-M, Xu W-B et al. Chinese guidelines for the diagnosis and treatment of hand, foot and mouth disease (2018 edition). World J Pediatr. 2018;14(5):437-47. doi:10.1007/s12519-018-0189-8.

21. Hubiche T, Schuffenecker I, Boralevi F, Léauté-Labrèze C, Bornebusch L, Chiaverini C et al. Dermatological spectrum of hand, foot and mouth disease from classical to generalized exanthema. Pediatr Infect Dis J. 2014;33(4):e92-e8. doi:10.1097/INF.0000000000000120.

22. Oberste MS, Peñaranda S, Rogers SL, Henderson E, Nix WA. Comparative evaluation of Taqman real-time PCR and semi-nested VP1 PCR for detection of enteroviruses in clinical specimens. J Clin Virol. 2010;49(1):73-4. doi:10.1016/j.jcv.2010.06.022.

23. Oberste MS, Maher K, Williams AJ, Dybdahl-Sissoko N, Brown BA, Gookin MS et al. Species-specific RT-PCR amplification of human enteroviruses: a tool for rapid species identification of uncharacterized enteroviruses. J Gen Virol. 2006;87(Pt 1):119-28.

24. Kroneman A, Vennema H, Deforche K, v d Avoort H, Peñaranda S, Oberste MS et al. An automated genotyping tool for enteroviruses and noroviruses. J Clin Virol. 2011;51(2):121-5. doi:10.1016/j.jcv.2011.03.006.

25. Edgar RC. MUSCLE: multiple sequence alignment with high accuracy and high throughput. Nucleic Acids Res. 2004;32(5):1792-7.

26. Edgar RC. MUSCLE: a multiple sequence alignment method with reduced time and space complexity. BMC bioinformatics. 2004;5:113.

27. Stamatakis A. RAxML version 8: a tool for phylogenetic analysis and post-analysis of large phylogenies. Bioinformatics. 2014;30(9):1312-3. doi:10.1093/bioinformatics/btu033 \%J Bioinformatics.

28. Goss EM, Tabima JF, Cooke DEL, Restrepo S, Fry WE, Forbes GA et al. The Irish potato famine pathogen Phytophthora infestans originated in central Mexico rather than the Andes. Proc Natl Acad Sci U S A. 2014;111(24):8791-6. doi:10.1073/pnas.1401884111.

29. Kumar S, Stecher G, Tamura K. MEGA7: Molecular Evolutionary Genetics Analysis Version 7.0 for Bigger Datasets. Mol Biol Evol. 2016;33(7):1870-4. doi:10.1093/molbev/msw054.

30. Oberste MS, Maher K, Kilpatrick DR, Flemister MR, Brown BA, Pallansch MA. Typing of human enteroviruses by partial sequencing of VP1. J Clin Microbiol. 1999;37(5):1288-93.

31. Oberste MS, Maher K, Kilpatrick DR, Pallansch MA. Molecular evolution of the human enteroviruses: correlation of serotype with VP1 sequence and application to picornavirus classification. J Virol. 1999;73(3):1941-8.

32. Mulders MN, Salminen M, Kalkkinen N, Hovi T. Molecular epidemiology of coxsackievirus B4 and disclosure of the correct VP1/2A(pro) cleavage site: evidence for high genomic diversity and long-term endemicity of distinct genotypes. J Gen Virol. 2000;81(Pt 3):803-12.

33. Hall TA. BioEdit : a user-friendly biological sequence alignment editor and analysis program for Windows 95/98/NT. Nucl Acids Symp Ser. 1999. 
34. Suchard MA, Lemey P, Baele G, Ayres DL, Drummond AJ, Rambaut A. Bayesian phylogenetic and phylodynamic data integration using BEAST 1.10. Virus Evol. 2018;4(1):vey016. doi:10.1093/ve/vey016.

35. Drummond AJ, Suchard MA, Xie D, Rambaut A. Bayesian phylogenetics with BEAUti and the BEAST 1.7. Mol Biol Evol. 2012;29(8):1969-73. doi:10.1093/molbev/mss075.

36. Drummond AJ, Rambaut A. BEAST: Bayesian evolutionary analysis by sampling trees. BMC Evol Biol. $2007 ; 7: 214$.

37. Rambaut A, Drummond AJ, Xie D, Baele G, Suchard MA. Posterior Summarization in Bayesian Phylogenetics Using Tracer 1.7. Syst Biol. 2018;67(5):901-4. doi:10.1093/sysbio/syy032.

38. Drummond AJ, Rambaut A, Shapiro B, Pybus OG. Bayesian coalescent inference of past population dynamics from molecular sequences. Mol Biol Evol. 2005;22(5):1185-92.

39. Rao DC, Ananda Babu M, Raghavendra A, Dhananjaya D, Kumar S, Maiya PP. Non-polio enteroviruses and their association with acute diarrhea in children in India. Infect Genet Evol. 2013;17:153-61. doi:10.1016/j.meegid.2013.04.011.

40. Ji H, Fan H, Lu P-X, Zhang X-F, Ai J, Shi C et al. Surveillance for severe hand, foot, and mouth disease from 2009 to 2015 in Jiangsu province: epidemiology, etiology, and disease burden. BMC Infect Dis. 2019;19(1):79. doi:10.1186/s12879-018-3659-7.

41. Wang ZL, Xia AM, Li YF, Su HL, Zhan LW, Chen YP et al. Socioeconomic burden of hand, foot and mouth disease in children in Shanghai, China. Epidemiol Infect. 2016;144(1):138-43. doi:10.1017/S0950268815001569.

42. Nhan LNT, Turner HC, Khanh TH, Hung NT, Lien LB, Hong NTT et al. Economic Burden Attributed to Children Presenting to Hospitals With Hand, Foot, and Mouth Disease in Vietnam. Open Forum Infect Dis. 2019;6(7). doi:10.1093/ofid/ofz284.

43. Ji T, Han T, Tan X, Zhu S, Yan D, Yang Q et al. Surveillance, epidemiology, and pathogen spectrum of hand, foot, and mouth disease in mainland of China from 2008 to 2017. Biosaf Health. 2019;1(1):32-40. doi:10.1016/j.bsheal.2019.02.005.

44. Zhang Y, Wang D, Yan D, Zhu S, Liu J, Wang H et al. Molecular evidence of persistent epidemic and evolution of subgenotype B1 coxsackievirus A16-associated hand, foot, and mouth disease in China. J Clin Microbiol. 2010;48(2):619-22. doi:10.1128/JCM.02338-09.

45. Bian L, Wang Y, Yao X, Mao Q, Xu M, Liang Z. Coxsackievirus A6: a new emerging pathogen causing hand, foot and mouth disease outbreaks worldwide. Expert Rev Anti Infect Ther. 2015;13(9):1061-71. doi:10.1586/14787210.2015.1058156.

46. Bian L, Gao F, Mao Q, Sun S, Wu X, Liu S et al. Hand, foot, and mouth disease associated with coxsackievirus A10: more serious than it seems. Expert Rev Anti Infect Ther. 2019;17(4):233-42. doi:10.1080/14787210.2019.1585242.

47. Zhang Y, Zhu Z, Yang W, Ren J, Tan X, Wang Y et al. An emerging recombinant human enterovirus 71 responsible for the 2008 outbreak of hand foot and mouth disease in Fuyang city of China. Virol J. 2010;7:94. doi:10.1186/1743422X-7-94.

48. Tian X, Zhang Y, Gu S, Fan Y, Sun Q, Zhang B et al. New coxsackievirus B4 genotype circulating in Inner Mongolia Autonomous Region, China. PLoS One. 2014;9(3):e90379. doi:10.1371/journal.pone.0090379.

\section{Figures}




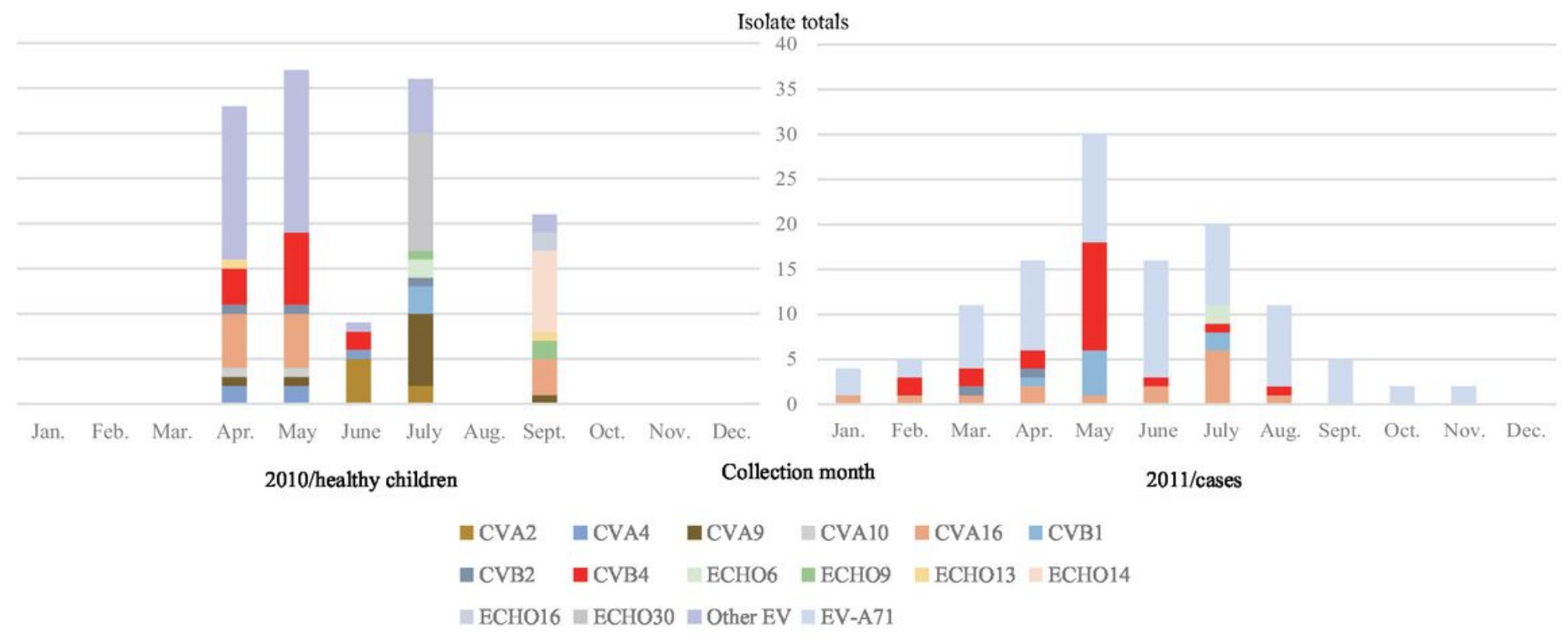

Figure 1

Pathogenic spectrum based on specimens from healthy humans in 2010 and from patients with HFMD in 2011 The abscissa indicates collection month, and the ordinate indicates isolate totals. Different pathogens are represented by different colors. 


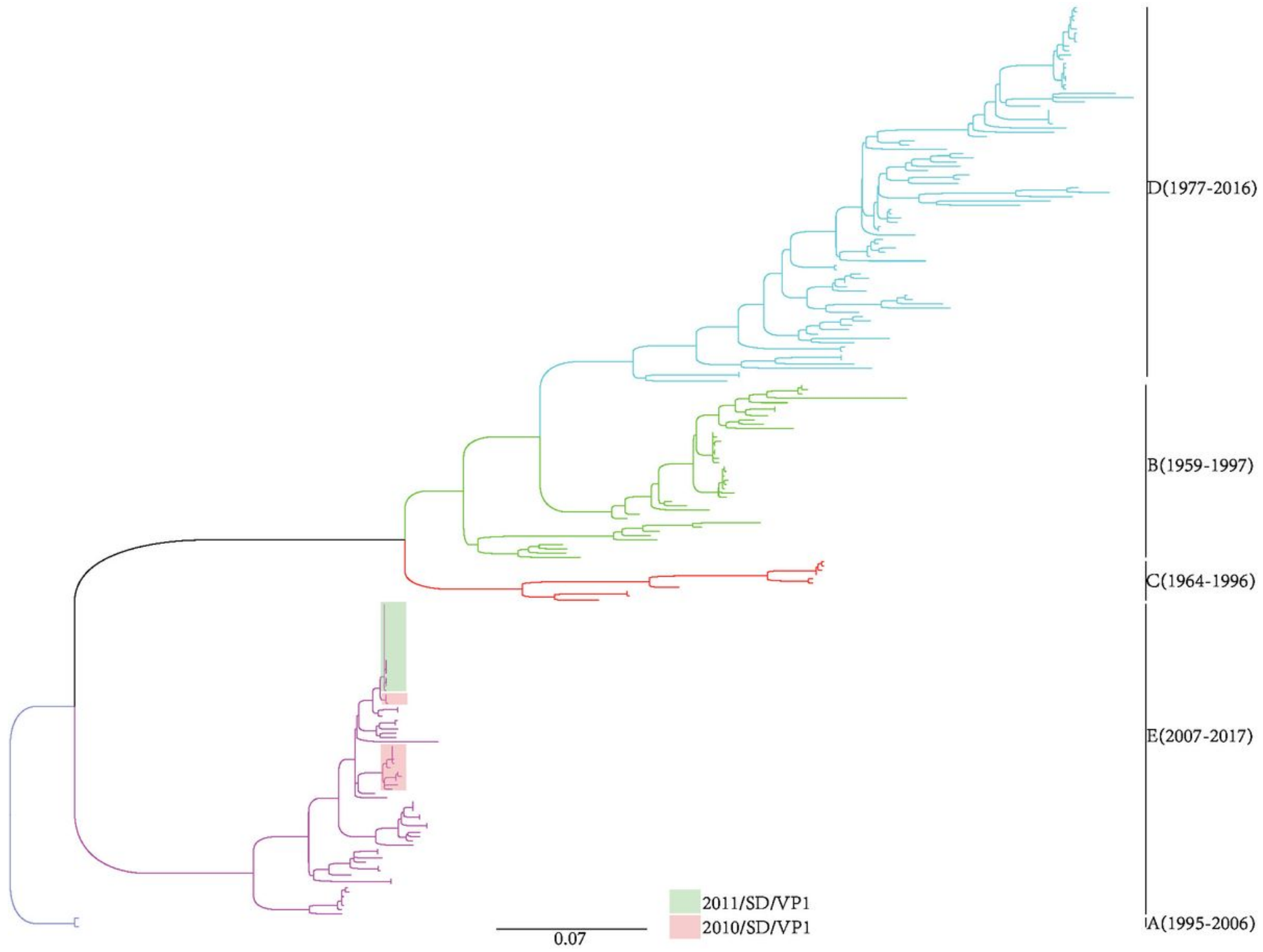

Figure 2

Maximum likelihood phylogenetic tree based on entire VP1 sequences (852 nt) Lines of different colors represent different genotypes. Scale bars represent substitutions per site per year. 


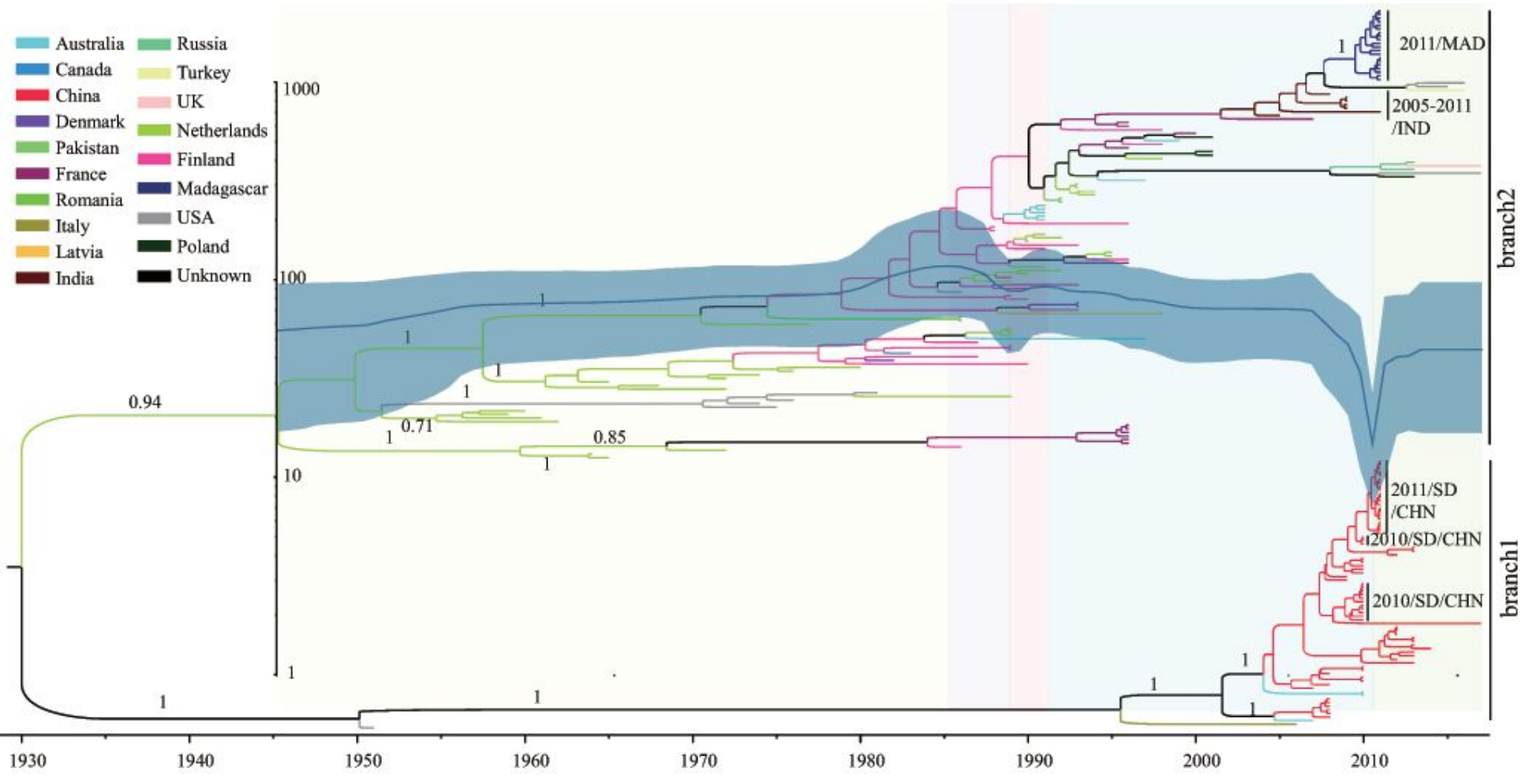

Figure 3

Bayesian maximum clade credibility (MCC) tree and Bayesian skyline plot constructed based on the entire VP1 sequence of CVB4 isolated worldwide ( $\mathrm{N}=200)(1)$ The MCC tree was obtained using an uncorrelated relaxed molecular clock model (Exponential) and exponential growth tree Prior. (2) The Bayesian skyline plot was based on the strict clock model. (3) Branches 1 and 2 represent two distinct lineages of CVB4.

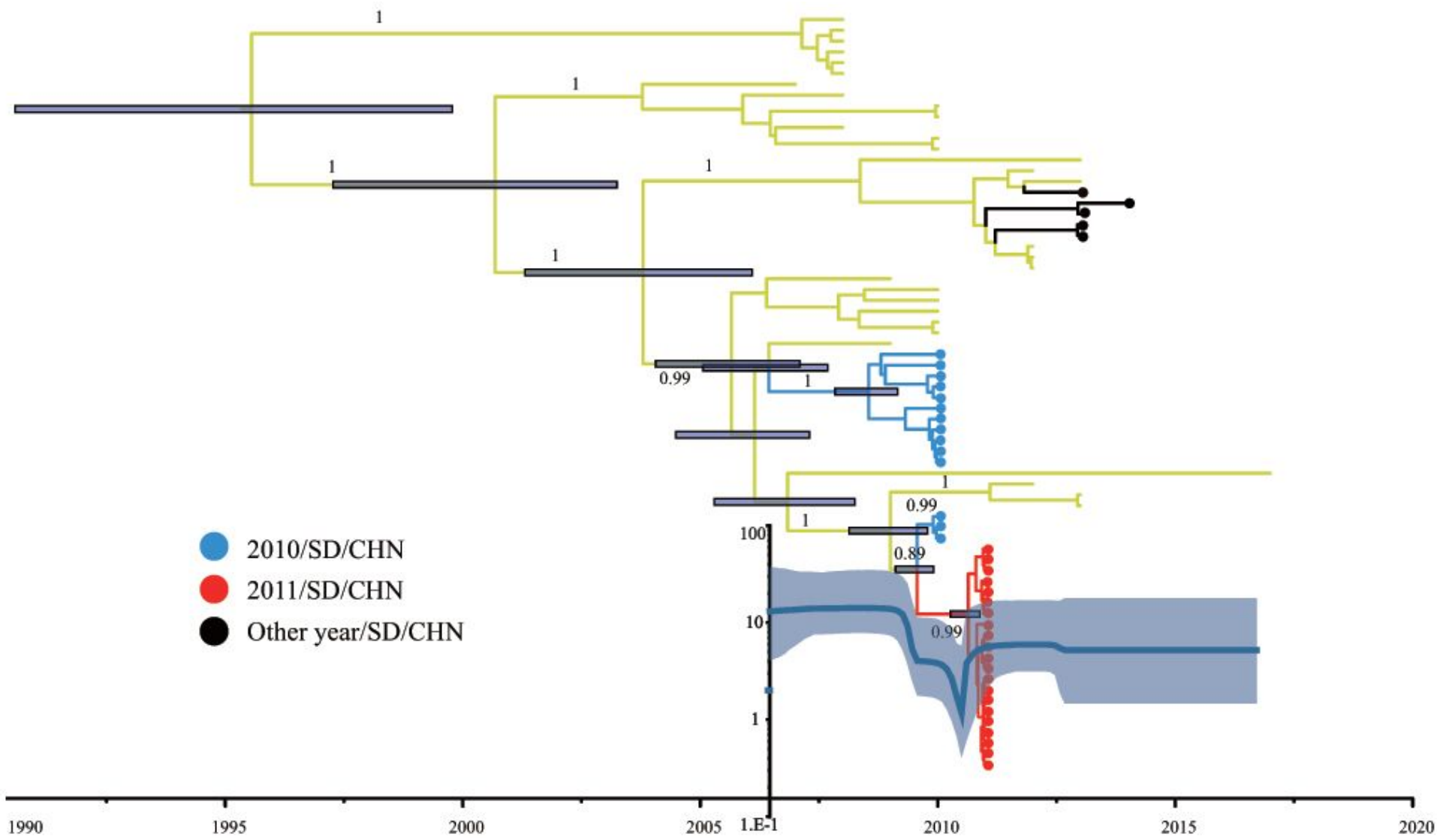




\section{Figure 4}

Bayesian maximum clade credibility (MCC) tree and Bayesian skyline plot based on entire VP1 sequences of CVB4 isolated in China ( $N=70)$ and the geographical distribution of Shandong CVB4 sequences The MCC tree and Bayesian skyline plot were obtained using the strict molecular clock model and Bayesian skyline tree prior. Blue marks indicate CVB4 strains isolated in Shandong Province in 2010, red marks indicate CVB4 strains isolated in Shandong Province in 2011, and black indicates CVB4 strains isolated in Shandong Province in other years. 